\title{
Grußwort Keine Angst vor „Diversity“
}

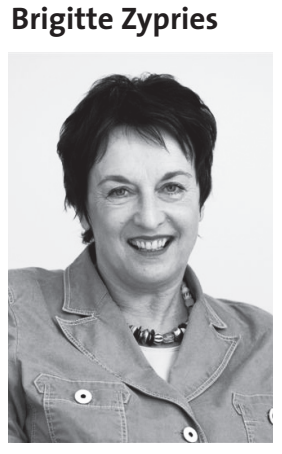

Brigitte Zypries, MdB Bundesministerin der Justiz, Berlin/Darmstadt
Erfurt ist ein gutes Pflaster für Juristinnen. Hier amtiert nicht nur Ingrid Schmidt als Präsidentin des Bundesarbeitsgerichts, in dieser Stadt geben Frauen auch in anderen Zweigen der Rechtspflege den Ton an: Sie stehen an den Spitzen von Landgericht, Amtsgericht und Anwaltsverein. Dies zeigt, dass der Deutsche Juristinnenbund seinen Tagungsort gut gewählt hat und deshalb bin ich besonders gern zu Ihnen nach Erfurt gekommen.

Der lokale Befund über Frauen in der Justiz ist zum Glück kein Einzelfall mehr. Er ist der Ausdruck einer guten Entwicklung, die wir schon seit Jahren beobachten können. Heute sind gut ein Drittel aller Anwälte und Richter in Deutschland Frauen. Dies ist ein Trend, der sich übrigens auch in der personellen Zusammensetzung des Bundesjustizministeriums niederschlägt. Etwa 50 Prozent aller Referentenstellen sind bei uns mit Frauen besetzt und Frauen stellen inzwischen auch ein Drittel aller Referatsleiterposten - Tendenz steigend. Eine Aufgabe, die vor wenigen Wochen zum ersten Mal in die Hand einer Frau übergegangen ist, ist die Präsidentschaft des Bundesverwaltungsgerichts. Marion Eckertz-Höfer ist nicht nur djbMitglied, sondern heute auch unter uns, und ich gratuliere ihr auch von dieser Stelle aus noch einmal vielmals zur Übernahme dieses wichtigen Amtes.

Die Entwicklung, die ich eben beschrieben habe, wird manchmal auf die Formel gebracht: Die Justiz in Deutschland wird weiblich. Das klingt ganz gut, aber richtig ist das nicht. Richtig ist vielmehr: Die Justiz wird repräsentativ! In einer Gesellschaft, in der 51 Prozent Frauen leben, kann es auf Dauer nicht gut sein, wenn die Justiz eine von Männern dominierte Staatsgewalt bliebe. Das ist nicht gut für die Rechtsfindung und es schadet auch sonst der Arbeit. Eine Justiz, die repräsentativ sein soll, braucht daher auch eine ausgewogene Geschlechterrepräsentanz. Inzwischen sind wir hier auf einem sehr guten Wege, und das liegt nicht zuletzt daran, dass wir die Angebote an Teilzeitarbeit, job-sharing oder Telearbeit deutlich ausgeweitet haben. Alles dies hilft, um Familie und Beruf besser unter einen Hut zu bekommen, denn auch wenn dieses Thema nicht nur Frauen angeht: In vielen Fällen ist die mangelnde Vereinbarkeit von Familie und Beruf noch immer der Grund, warum Frauen auf eine Berufstätigkeit verzichten oder ihnen der Aufstieg in Führungspositionen verwehrt wird. Tatsache ist aber auch, dass die Justiz wiederum leider nicht repräsentativ ist für die gesamte Gesellschaft. Das, was die Justiz geschafft hat, ist noch längst nicht der Regelfall.

Es gibt nach wie vor Berufsfelder - auch solche, in denen Juristinnen aktiv sind - in denen es an Chancengleichheit und einer angemessenen Repräsentanz von Frauen fehlt. Bei Führungsposten in der Wirtschaft kommen Frauen nach wie vor zu kurz. Die Zahl von Frauen in Vorständen oder Aufsichtsräten ist in Deutschland - auch im internationalen Vergleich - außerordentlich gering. Der Juristinnenbund hat sich vorgenommen, hieran etwas zu ändern, und ich unterstütze dieses Vorhaben sehr. Am 18. Oktober veranstaltet der djb in Berlin das Frauendinner Corporate Governance zum Thema Frauen in Aufsichtsräten. Voraussetzung dafür, dass sich hier etwas ändert, ist nicht zuletzt ein Mentalitätswechsel, und das berührt ein Thema, das im Mittelpunkt dieses Kongresses stehen soll: Diversity.

Für die juristische Normalverbraucherin ist es nicht ganz einfach, mit den Begrifflichkeiten zu hantieren, die die internationale Debatte hier hervorbringt: Erst „Gender Mainstreaming“, jetzt „Diversity“. Niemand sollte sich davon abschrecken lassen, denn der Gedanke von „Diversity“ ist bestechend. Es geht um die ökonomischen Aspekte von Gleichstellung und Diskriminierungsschutz, und das heißt auch, mit den Kräften des Marktes gesellschaftspolitische Ziele zu erreichen. Der Gedanke der Vielfalt umfasst hier allerdings mehr als nur das Geschlecht. Er bezieht auch Alter, Herkunft oder sexuelle Orientierung mit ein. Dass eine innovative Personalstrategie, die solche Eigenschaften von Beschäftigten in den Blick nimmt, wirtschaftlich sinnvoll sein kann, liegt auf der Hand:

Fortschritt und Wachstum brauchen Inspiration und neue Ideen. Die werden aber nicht im Mainstream geboren, sondern nur dort, wo Vielfalt und Dynamik herrschen. Eine bunte Truppe macht deshalb mehr Sinn als eine Belegschaft in grauer Uniformität.

Je internationaler unser Wirtschaftsleben wird, umso wichtiger sind auch Mitarbeiterinnen, die 




Präsidentin Jutta Wagner überreicht dem Thüringer Justizminister

Harald Schliemann das vom djb herausgegebene Buch „Juristinnen - Lexikon zu Leben und Werk“. Foto: CL.

sich in der Welt auskennen. Man wird kein Global Player, wenn man nur Spieler aus der Provinz hat. Wie wichtig so etwas ist, habe ich während der deutschen EU-Ratspräsidentschaft erlebt. Die Mitarbeiterinnen und Mitarbeiter des Justizministeriums mit bi-nationalen Wurzeln waren in dieser Zeit ein ganz besonderer Gewinn.

Vielfalt statt Homogenität kann auch für mehr Flexibilität sorgen. Für manche Personalabteilung gelten Homosexuelle inzwischen ja als ideale Mitarbeiter. Die können Überstunden machen, ohne dass zu Hause eine vielköpfige Familie murrt, und ihren Urlaub müssen sie auch nicht alle gleichzeitig während der Schulferien nehmen.

Ob solche Bilder tatsächlich stimmen, kann man bezweifeln, schließlich gibt es inzwischen immer mehr sogenannte Regenbogenfamilien. Tatsache aber bleibt, dass der DiversityGedanke außerordentlich reizvoll ist: Chancengleichheit wird hier nicht durch Gesetze, Quoten und Verbote erreicht, sondern durch die Kräfte des Marktes. Weil die Unternehmen das Prinzip „Wertschöpfung durch Vielfalt“ entdecken, sorgen sie selbst dafür, dass niemand diskriminiert wird. Sie erkennen, dass Chancengleichheit und Toleranz keine großmütig gewährte Gunst sind, sondern eine ökonomische Notwendigkeit, um sich im Wettbewerb zu behaupten. Ob dieser Ansatz in der Praxis wirklich funktioniert, bleibt abzuwarten. Kann die unsichtbare Hand des Marktes die Kraftprobe mit Vorurteilen und dem old-boys-network wirklich gewinnen? Eine endgültige Antwort steht noch aus, aber die Erfahrungsberichte, die für morgen geplant sind, werden bestimmt spannend.

So reizvoll der Diversity-Ansatz ist, so weiß ich doch auch, dass viele von Ihnen die Sache mit etwas gemischten Gefühlen sehen. Viele Frauen haben die Sorge, dass durch ein Mehr an Aufmerksamkeit für anders definierte Gruppen die Frau- enförderung zu kurz kommen könnte. Ich meine, diese Sorge ist unbegründet. Unser Ziel muss Chancengleichheit für alle und die Beseitigung jeder Form von Diskriminierung sein. Nicht gruppenbezogene Vorurteile, sondern die persönliche Leistung und die individuellen Stärken sollen für Personalentscheidungen maßgebend sein. Chancengleichheit und Leistungsgerechtigkeit sind für mich zwei Seiten einer Medaille. Ich denke, wir wissen alle, was wir Frauen leisten können, und deshalb sehe ich keinen Grund, warum wir den Diversity-Gedanken fürchten sollten.

Es steht außer Frage, dass wir auch künftig weitere Anstrengungen in Politik, Wirtschaft und Gesellschaft brauchen, um die Gleichstellung von Frauen zu verwirklichen. Der Deutsche Juristinnenbund ist seit jeher eine starke Triebfeder der Entwicklung und vieles von dem, was in der Vergangenheit erreicht wurde, ist nicht zuletzt der Verdienst der Frauen im djb. Mit der Reform des Unterhaltsrechts, dem Güterrecht oder der Strukturreform im Versorgungsausgleich arbeiten wir derzeit an drei Gesetzesprojekten, bei denen es nicht zuletzt darum geht, die Selbstbestimmung der Frauen zu stärken. Der Deutsche Juristinnenbund hat mit vielen Stellungnahmen die Arbeit an diesen Projekten immer wieder entscheidend vorangebracht. Ich möchte deshalb zum Abschluss Dank sagen. Dank an alle Kolleginnen, die sich im djb, in seinen Gliederungen und seinen Kommissionen engagieren. Ihr und Euer Einsatz ist auch eine wichtige Unterstützung für all jene, die sich in der Politik für die Belange von Frauen stark machen. Mit Ihrer Arbeit tragen Sie ganz entscheidend dazu bei, dass unser Staat niemals nachlässt seinen Verfassungsauftrag zu erfüllen: nämlich die Durchsetzung der Gleichberechtigung zu fördern und auf die Beseitigung bestehender Nachteile hinzuwirken. 